\title{
Eigenvalue problem for fractional differential equations with nonlinear integral and disturbance parameter in boundary conditions
}

\author{
Wenxia Wang ${ }^{1 *}$ and Xiaotong Guo ${ }^{2}$
}

"Correspondence:
wwxgg@126.com
'Department of Mathematics,
Taiyuan Normal University, Taiyuan,
030012, P.R. China
Full list of author information is
available at the end of the article

available at the end of the article

\begin{abstract}
This paper is concerned with the existence, nonexistence, uniqueness, and multiplicity of positive solutions for a class of eigenvalue problems of nonlinear fractional differential equations with a nonlinear integral term and a disturbance parameter in the boundary conditions. By using fixed point index theory we give the critical curve of eigenvalue $\lambda$ and disturbance parameter $\mu$ that divides the range of $\lambda$ and $\mu$ for the existence of at least two, one, and no positive solutions for the eigenvalue problem. Furthermore, by using fixed point theorem for a sum operator with a parameter we establish the maximum eigenvalue interval for the existence of the unique positive solution for the eigenvalue problem and show that such a positive solution depends continuously on the parameter $\lambda$ for given $\mu$. In particular, we give estimates for the critical value of parameters. Two examples are given to illustrate our main results.
\end{abstract}

MSC: 34B18; 34B15

Keywords: integral boundary value problem; eigenvalue problem; disturbance parameter; Caputo fractional derivative; positive solution; cone

\section{Introduction and preliminaries}

Fractional differential equations have been extensively investigated in recent years, due to a wide range of applications in various fields of sciences and engineering such as control, porous media, electromagnetic, and so forth; see [1-18] and the references therein. Since the integral boundary value problems can better describe the actual phenomenon, the existence of positive solutions for fractional integral boundary value problems has attracted considerable attention, and fruits from research into it emerge continuously. For a small sample of such a work, we refer the reader to [19-27] and the references therein.

On the other hand, the eigenvalue problems are one of the most active fields in differential equation theories, and the eigenvalue problems of nonlinear fractional differential equations have been concerned by some authors; see [28-31]. Recently, [32] and [33] studied the fractional eigenvalue problems with integral boundary conditions and obtain some interesting results. By the Guo-Krasnoselskii fixed point theorem Wang et al. [33] investigated the eigenvalue interval for the existence and nonexistence of at least one positive

(c) 2016 Wang and Guo. This article is distributed under the terms of the Creative Commons Attribution 4.0 International License (http://creativecommons.org/licenses/by/4.0/), which permits unrestricted use, distribution, and reproduction in any medium, provided you give appropriate credit to the original author(s) and the source, provide a link to the Creative Commons license, and indicate if changes were made. 
solution for the following eigenvalue problem:

$$
\left\{\begin{array}{l}
{ }^{C} D_{0^{+}}^{\alpha} x(t)+\lambda f(t, x(t))=0, \quad 0<t<1, n<\alpha \leq n+1, n \geq 2, n \in N, \\
x(0)=x^{\prime \prime}(0)=x^{\prime \prime \prime}(0)=\cdots=x^{(n)}(0)=0, \\
x(1)=\xi \int_{0}^{1} x(s) d s,
\end{array}\right.
$$

where $0<\xi<2$ and $f \in C\left([0,1] \times R_{+}, R_{+}\right)$. At the same time, when we apply the methods of the differential equations to solve actual problems, it is inevitable that there always exists disturbance that has great influence on the existence of solutions. Jia and Liu [34] studied the following boundary value problem:

$$
\left\{\begin{array}{l}
-{ }^{C} D_{0^{+}}^{\alpha} x(t)=f(t, x(t)), \quad 0<t<1, \\
m_{1} x(0)-n_{1} x^{\prime}(0)=0 \\
m_{2} x(1)+n_{2} x^{\prime}(1)=\int_{0}^{1} k(s) x(s) d s+a,
\end{array}\right.
$$

where $1<\alpha \leq 2, f \in C\left([0,1] \times R_{+}, R_{+}\right), m_{i}, n_{i} \geq 0, m_{i}^{2}+n_{i}^{2}>0, i=1,2, k \in C\left(R_{+}, R_{+}\right), a \in R_{+}$. They discussed the impact of disturbance parameters $a$ on the existence of positive solutions by the method of upper and lower solutions, fixed point index theory, and the Schauder fixed point theorem. Given the above, it is worthwhile to study the eigenvalue problem of nonlinear fractional differential equations with nonlinear integral and disturbance parameter in the boundary conditions. To the best of authors' knowledge, there are few papers reported on this topic.

In this paper, we will study the following eigenvalue problem of a fractional differential equation (FEP):

$$
\left\{\begin{array}{l}
{ }^{C} D_{0^{+}}^{\alpha} x(t)+\lambda f(t, x(t))=0, \quad 0<t<1, \\
a x(0)-b x^{\prime}(0)=0, \\
x(1)=\int_{0}^{1} k(s) g(x(s)) d s+\mu,
\end{array}\right.
$$

where ${ }^{C} D_{0^{+}}^{\alpha}$ is the Caputo fractional derivative of order $\alpha, 1<\alpha \leq 2, \lambda, \mu \geq 0$. Throughout this paper, we assume that $f \in C\left([0,1] \times R_{+}, R_{+}\right), g \in C\left(R_{+}, R_{+}\right), k \in C\left([0,1], R_{+}\right), k \not \equiv 0$, $R_{+}=[0,+\infty), a, b \in R_{+}, a+b>0$, and $\frac{a}{a+b}<\alpha-1$.

The FEP (1) seems to be studied for the first time. The purpose of this paper is to find the critical curve of parameters $\lambda$ and $\mu$ dividing the range of $\lambda$ and $\mu$ for the existence of at least two, one, and no positive solutions and to establish the maximum eigenvalue interval for the existence of the unique positive solution for the eigenvalue problem. The main tools used in this paper are fixed point index theory, the fixed point theorem of a sum operator with a parameter, and a sufficient and necessary condition for the existence of a fixed point for a concave operator. In particular, the positivity of a solution $x(t)$ of FEP (1) means that $x(t) \geq 0$ for $t \in[0,1]$ and $x(t)>0$ for $t \in(0,1)$.

The paper is organized as follows. In Section 2, we establish an operator equation that is equivalent to FEP (1) and present properties of solutions of FEP (1). In Section 3, we obtain the critical curve of $\lambda$ and $\mu$ and establish an eigenvalue interval for the existence of at least two, one, and no positive solutions for FEP (1) according to the range of the disturbance parameter $\mu$. These results show that the impact of the parameter $\mu$ on the eigenvalue interval for the existence of positive solutions and on their number. In Section 4, under 
some mild assumption, we establish the maximum eigenvalue interval for the existence of the unique positive solution for FEP (1) and show that such a positive solution depends continuously on the parameter $\lambda$. In particular, we give estimates for the critical values of parameters. Finally, two examples are given to illustrate our main results.

For convenience of the readers, we first present some basic notation and results that will be used in the proofs of our theorems. We refer to [1-4] for details.

Definition 1.1 Let $x:(0,+\infty) \rightarrow R$ be a function, and $\alpha>0$. The Riemann-Liouville fractional integral of order $\alpha$ of $x$ is defined by

$$
I_{0^{+}}^{\alpha} x(t)=\frac{1}{\Gamma(\alpha)} \int_{0}^{t}(t-s)^{\alpha-1} x(s) d s
$$

provided that the integral exists. The Caputo fractional derivative of order $\alpha$ of $x$ is defined by

$$
{ }^{C} D_{0^{+}}^{\alpha} x(t)=\frac{1}{\Gamma(n-\alpha)} \int_{0}^{t}(t-s)^{n-\alpha-1} x^{(n)}(s) d s,
$$

provided that the right side is pointwise defined on $(0,+\infty)$, where $n=[\alpha]+1, n-1<\alpha<n$, and $\Gamma$ denotes the gamma function. If $\alpha=n$, then ${ }^{C} D_{0^{+}}^{\alpha} x(t)=x^{(n)}(t)$.

Lemma 1.2 If $x \in A C^{n}[0,1]$, then the Caputo fractional derivative ${ }^{C} D_{0^{+}}^{\alpha} x(t)$ exists almost everywhere on $[0,1]$, where $A C^{n}[0,1]=\left\{x \in C^{n-1}[0,1] \mid x^{(n-1)}\right.$ is absolutely continuous $\}$, and $n$ is the smallest integer greater than or equal to $\alpha$.

Lemma 1.3 If $x \in C^{n}[0,1]$, then

$$
I_{0^{+}}^{\alpha}{ }^{C} D_{0^{+}}^{\alpha} x(t)=x(t)+C_{0}+C_{1} t+C_{1} t^{2}+\cdots+C_{n-1} t^{n-1},
$$

where $n$ is the smallest integer greater than or equal to $\alpha$.

In the rest of this section, we present some notation and some known results on cone theory. We refer to [35, 36], and [37] for details.

Let $E$ be a real Banach space partially ordered by a cone $P \subset E$, that is, $x \leq y$ iff $y-x \in P$. If $x \leq y$ and $x \neq y$, then we write $x<y$ or $y>x$. By $\theta$ we denote the zero element of $E$. A cone $P$ is said to be normal if there exists a positive number $N$, called the normal constant of $P$, such that $\theta \leq x \leq y$ implies $\|x\| \leq N\|y\|$. For $u, v \in E, u \leq v$, denote $[u, v]=\{x \in E \mid$ $u \leq x \leq v\}$.

Given $e>0$ (i.e., $e \in P$ and $e \neq \theta$ ), set

$$
\begin{aligned}
& P_{e}=\left\{x \in E \mid \text { there exist } l_{1}=l_{1}(x)>0, l_{2}=l_{2}(x)>0 \text { such that } l_{1} e \leq x \leq l_{2} e\right\}, \\
& P_{e}^{*}=\{x \in E \mid \text { there exists } l=l(x)>0 \text { such that } 0 \leq x \leq l e\},
\end{aligned}
$$

then $P_{e} \subset P_{e}^{*} \subset P$.

Let $D \subset E$. An operator $T: D \rightarrow E$ is said to be increasing if for $x, y \in D, x \leq y \Rightarrow T x \leq T y$. An element $x^{*} \in D$ is called a fixed point of $T$ if $T x^{*}=x^{*}$. 
Lemma 1.4 ([35, 36]) Let $P$ be a cone of $E$, and $\Omega$ be a bounded open subset in $E$ with $\theta \in \Omega$. Assume that $T: P \cap \bar{\Omega} \rightarrow P$ is a completely continuous operator.

(i) If $T x \neq \rho x$ for $x \in P \cap \partial \Omega$ and $\rho \geq 1$, then $i(T, P \cap \Omega, P)=1$.

(ii) If $T x \neq x$ and $\|T x\| \geq\|x\|$ for $x \in P \cap \partial \Omega$, then $i(T, P \cap \Omega, P)=0$.

Lemma 1.5 ([37]) Let $P$ be a normal cone of $E, A, T: P \rightarrow P$ be increasing and satisfy

(G1) $A\left(P_{e}\right) \subset P_{e}^{*}$ and $A(r x) \geq r A x$ for $x \in P_{e}$ and $r \in(0,1)$;

(G2) $T\left(P_{e}\right) \subset P_{e}$, and there exists $\tau \in(0,1)$ such that $T(r x) \geq r^{\tau} T x$ for $x \in P_{e}, r \in(0,1)$.

Then there exists $\lambda^{*}>0$ such that $\lambda A+T$ has a unique fixed point $x_{\lambda} \in P_{e}$ for $\lambda \in\left[0, \lambda^{*}\right)$ and has no fixed point in $P_{e}$ for $\lambda \geq \lambda^{*}$. Moreover, such a fixed point $x_{\lambda}$ has the following properties:

(i) the recurrent sequence $u_{n}=\lambda A u_{n-1}+T u_{n-1}(n=1,2, \ldots)$ for any $u_{0} \in P_{e}$ converges to $x_{\lambda}$, that is, $\lim _{n \rightarrow+\infty}\left\|u_{n}-x_{\lambda}\right\|=0$;

(ii) $x_{\lambda}$ is increasing in $\lambda$ for $\lambda \in\left[0, \lambda^{*}\right)$;

(iii) $x_{\lambda}$ is continuous with respect to $\lambda$ for $\lambda \in\left[0, \lambda^{*}\right)$.

Lemma 1.6 ([37]) Let $P$ be a normal cone in $E$, and $T: P \rightarrow P$ be an increasing operator. Suppose that $T\left(P_{e}\right) \subset P_{e}$ and for any $r \in(0,1)$ and $[y, z] \subset P_{e}$, there exists $\eta(r, y, z)>0$ such that

$$
T(r x) \geq r(1+\eta(r, y, z)) T x, \quad \forall x \in[y, z], r \in(0,1) .
$$

Then $T$ has a unique fixed point $x^{*}$ in $P_{e}$ if and only if there exist $u, v \in P_{e}$ such that $u \leq$ $T u \leq T v \leq v$. Moreover, for any initial value $u_{0} \in P_{e}$ and the recurrent sequence $u_{n}=T u_{n-1}$ $(n=1,2, \ldots)$, we have $\lim _{n \rightarrow+\infty}\left\|u_{n}-x^{*}\right\|=0$.

\section{Properties of positive solutions and equivalent operator equation}

In this section, we will apply Lemma 1.3 to present the existence and uniqueness results for a solution of a linear fractional boundary value problem; moreover, we present the operator equation equivalent to FEP (1). This is important for our research.

We set $E=C[0,1]$, the Banach space of all continuous functions on $[0,1]$ with the norm $\|x\|=\max \{|x(t)| \mid t \in[0,1]\}$. Let $P=\{x \in C[0,1] \mid x(t) \geq 0, t \in[0,1]\}$. It is clear that $P$ is a normal cone with normal constant 1 .

For $y, z \in C[0,1]$, consider the linear fractional boundary value problem (BVP)

$$
\left\{\begin{array}{l}
{ }^{C} D_{0^{+}}^{\alpha} x(t)+y(t)=0, \quad 0<t<1, \\
a x(0)-b x^{\prime}(0)=0, \\
x(1)=\int_{0}^{1} k(s) z(s) d s+\mu .
\end{array}\right.
$$

Lemma 2.1 BVP (2) has a unique solution

$$
x(t)=\int_{0}^{1} G(t, s) y(s) d s+\frac{a t+b}{a+b} \int_{0}^{1} k(s) z(s) d s+\frac{a t+b}{a+b} \mu,
$$

where

$$
G(t, s)=\frac{1}{\Gamma(\alpha)} \begin{cases}\frac{(a t+b)(1-s)^{\alpha-1}}{a+b}-(t-s)^{\alpha-1}, & 0 \leq s \leq t \leq 1, \\ \frac{(a t+b)(1-s)^{\alpha-1}}{a+b}, & 0 \leq t \leq s \leq 1 .\end{cases}
$$


Proof From Lemma 1.3 we have

$$
\begin{aligned}
& x(t)=-\frac{1}{\Gamma(\alpha)} \int_{0}^{t}(t-s)^{\alpha-1} y(s) d s+c_{0}+c_{1} t, \\
& x^{\prime}(t)=-\frac{1}{\Gamma(\alpha-1)} \int_{0}^{t}(t-s)^{\alpha-2} y(s) d s+c_{1} .
\end{aligned}
$$

By the boundary conditions in BVP (2) we get

$$
a c_{0}+b c_{1}=0, \quad-\frac{1}{\Gamma(\alpha)} \int_{0}^{1}(1-s)^{\alpha-1} y(s) d s+c_{0}+c_{1}=\int_{0}^{1} k(s) z(s) d s+\mu .
$$

Hence,

$$
\begin{aligned}
& c_{0}=\frac{b}{a+b}\left[\frac{1}{\Gamma(\alpha)} \int_{0}^{1}(1-s)^{\alpha-1} y(s) d s+\int_{0}^{1} k(s) z(s) d s+\mu\right], \\
& c_{1}=\frac{a}{a+b}\left[\frac{1}{\Gamma(\alpha)} \int_{0}^{1}(1-s)^{\alpha-1} y(s) d s+\int_{0}^{1} k(s) z(s) d s+\mu\right] .
\end{aligned}
$$

This means that

$$
x(t)=\int_{0}^{1} G(t, s) y(s) d s+\frac{a t+b}{a+b} \int_{0}^{1} k(s) z(s) d s+\frac{a t+b}{a+b} \mu .
$$

The proof is complete.

Lemma 2.2 The function $G(t, s)$ defined by (4) satisfies

$$
0 \leq G(t, s) \leq \frac{(a t+b)(1-s)^{\alpha-1}}{(a+b) \Gamma(\alpha)}, \quad t, s \in[0,1]
$$

and $\max _{t \in[0,1]} G(t, s)=G(s, s), s \in[0,1]$.

Proof From (4) we easily see that

$$
G(t, s) \leq \frac{(a t+b)(1-s)^{\alpha-1}}{(a+b) \Gamma(\alpha)}, \quad t, s \in[0,1]
$$

For $0 \leq s<t \leq 1$, by (4) we have

$$
\frac{\partial G(t, s)}{\partial t}=\frac{1}{\Gamma(\alpha)}\left(\frac{a(1-s)^{\alpha-1}}{a+b}-\frac{\alpha-1}{(t-s)^{2-\alpha}}\right) \leq \frac{(1-s)^{\alpha-1}}{\Gamma(\alpha)}\left(\frac{a}{a+b}-(\alpha-1)\right)<0,
$$

which implies that

$$
G(s, s)>G(t, s)>G(1, s)=0, \quad 0 \leq s<t<1 .
$$

So, the continuity of $G(t, s)$ leads to

$$
0 \leq G(t, s) \leq G(s, s), \quad 0 \leq s \leq t \leq 1
$$

It is clear by (4) that also $0 \leq G(t, s) \leq G(s, s)$ for $0 \leq t \leq s \leq 1$. This ends the proof. 
Lemma 2.3 Let $y, z \in C\left([0,1], R_{+}\right)$. Then, for arbitrary given $0<\sigma<1$, the unique solution $x(t)$ of $B V P(2)$ satisfies

$$
x(t) \geq \gamma e(t)\|x\|, \quad t \in[0, \sigma],
$$

where

$$
0<\gamma=\frac{a \sigma+b}{a+b}-\sigma^{\alpha-1}<1 \quad \text { and } \quad e(t)=\frac{a t+b}{a+b}, \quad t \in[0,1] .
$$

Proof By Lemmas 2.1 and 2.2 we have

$$
x(t) \leq e(t)\left[\frac{1}{\Gamma(\alpha)} \int_{0}^{1}(1-s)^{\alpha-1} y(s) d s+\int_{0}^{1} k(s) z(s) d s+\mu\right], \quad t \in[0,1] .
$$

Moreover,

$$
\|x\| \leq \frac{1}{\Gamma(\alpha)} \int_{0}^{1}(1-s)^{\alpha-1} y(s) d s+\int_{0}^{1} k(s) z(s) d s+\mu .
$$

For $0 \leq s \leq t \leq \sigma$, (5) implies that

$$
G(t, s) \geq G(\sigma, s)=\frac{1}{\Gamma(\alpha)}\left(\frac{a \sigma+b}{a+b}(1-s)^{\alpha-1}-(\sigma-s)^{\alpha-1}\right) .
$$

This, together with

$$
\frac{\partial G(\sigma, s)}{\partial s} \geq \frac{\alpha-1}{\Gamma(\alpha)}\left((\sigma-s)^{\alpha-2}-(1-s)^{\alpha-2}\right)>0,
$$

gives

$$
G(t, s) \geq G(\sigma, s) \geq G(\sigma, 0)=\frac{1}{\Gamma(\alpha)}\left(\frac{a \sigma+b}{a+b}-\sigma^{\alpha-1}\right)=\frac{\gamma}{\Gamma(\alpha)}>0
$$

and $0<\gamma<1$. From (3), (7), and (9) we have

$$
\begin{aligned}
x(t) & \geq \frac{\gamma}{\Gamma(\alpha)} \int_{0}^{t} y(s) d s+e(t)\left(\frac{1}{\Gamma(\alpha)} \int_{t}^{1}(1-s)^{\alpha-1} y(s) d s+\int_{0}^{1} k(s) z(s) d s+\mu\right) \\
& \geq \gamma e(t)\left(\frac{1}{\Gamma(\alpha)} \int_{0}^{1}(1-s)^{\alpha-1} y(s) d s+\int_{0}^{1} k(s) z(s) d s+\mu\right) \\
& \geq \gamma e(t)\|x\|, \quad t \in[0, \sigma] .
\end{aligned}
$$

This ends the proof.

Lemma 2.4 A function $x(t)$ is a solution of FEP (1) if and only if $x(t)$ is a solution of the integral equation

$$
x(t)=\lambda \int_{0}^{1} G(t, s) f(s, x(s)) d s+\frac{a t+b}{a+b}\left(\int_{0}^{1} k(s) g(x(s)) d s+\mu\right) .
$$


Proof According to Lemma 2.1, it is evident that the solution of FEP (1) is the solution of the integral equation (10).

On the other hand, if $x \in C[0,1]$ is the solution of (10), then

$$
\begin{aligned}
x^{\prime}(t)= & -\frac{\lambda}{\Gamma(\alpha-1)} \int_{0}^{t}(t-s)^{\alpha-2} f(s, x(s)) d s+\frac{a \lambda}{(a+b) \Gamma(\alpha)} \int_{0}^{1}(1-s)^{\alpha-1} f(s, x(s)) d s \\
& +\frac{a}{(a+b)}\left(\int_{0}^{1} k(s) g(x(s)) d s+\mu\right) .
\end{aligned}
$$

It is easy to see that $x^{\prime} \in A C[0,1]$ and $x \in A C^{2}[0,1]$. From Lemma 1.2 we obtain that ${ }^{C} D_{0^{+}}^{\alpha} x$ exists almost everywhere on $[0,1]$. Noting that

$$
x^{\prime \prime}(t)=-\frac{d}{d t}\left(\frac{\lambda}{\Gamma(\alpha-1)} \int_{0}^{t}(t-s)^{\alpha-2} f(s, x(s)) d s\right)=-\lambda \frac{d}{d t} I_{0^{+}}^{\alpha-1} f(t, x(t)),
$$

we can conclude that ${ }^{C} D_{0^{+}}^{\alpha} x(t)=-\lambda f(t, x(t))$ and $x$ is the solution of FEP (1). The proof is complete.

By Lemmas 2.3 and 2.4 we can get the following result.

Lemma 2.5 If $x \in P \backslash\{\theta\}$ is a solution of FEP $(1)$, then $x(t) \geq 0$ for $t \in[0,1]$ and $x(t)>0$ for $t \in(0,1)$, that is, $x(t)$ is a positive solution of FEP $(1)$.

For given $0<\sigma_{1}<\sigma_{2}<1$, let

$$
K=\left\{x \in P \mid x(t) \geq \gamma_{0} e(t)\|x\|, t \in\left[\sigma_{1}, \sigma_{2}\right]\right\},
$$

where $\gamma_{0}=\frac{a \sigma_{2}+b}{a+b}-\sigma_{2}^{\alpha-1}$, and $e(t)$ is defined as in Lemma 2.3. It is easy to show that $K \subset P$ is also a cone in $E$.

Define the operators $A, T_{\mu}, C_{(\lambda, \mu)}: P \rightarrow E$ by

$$
\begin{aligned}
& (A x)(t)=\int_{0}^{1} G(t, s) f(s, x(s)) d s, \\
& \left(T_{\mu} x\right)(t)=\left(\int_{0}^{1} k(s) g(x(s)) d s+\mu\right) e(t), \\
& \left(C_{(\lambda, \mu)} x\right)(t)=\lambda(A x)(t)+\left(T_{\mu} x\right)(t) .
\end{aligned}
$$

It is clear by Lemma 2.4 that $x$ is a solution of FEP (1) if and only if $C_{(\lambda, \mu)} x=x$.

Lemma 2.6 The operator $C_{(\lambda, \mu)}: P \rightarrow K$ is completely continuous.

Proof According to Lemma 2.3, it is easy to verify that $C_{(\lambda, \mu)}(P) \subset K$.

Let $D \subset P$ be a bounded set. Then there exists a constant $M>0$ such that $\|x\| \leq M$ for $x \in D$. Since $f$ and $g$ are continuous, there exists a constant $M_{1}>0$ such that

$$
\max _{t \in[0,1], \kappa \in[0, M]} f(t, \kappa) \leq M_{1}, \quad \max _{\kappa \in[0, M]} g(\kappa) \leq M_{1} .
$$


Hence, from Lemma 2.2 we have

$$
(A x)(t) \leq \frac{M_{1}}{\Gamma(\alpha)} \int_{0}^{1}(1-s)^{\alpha-1} d s, \quad\left(T_{\mu} x\right)(t) \leq M_{1} \int_{0}^{1} k(s) d s+\mu, \quad x \in D,
$$

that is, $A(D)$ and $T_{\mu}(D)$ are uniformly bounded.

It is clear that $G(t, s)$ is uniformly continuous on $[0,1] \times[0,1]$, which implies that for any $\epsilon>0$, there exists $\delta_{1}>0$ such that

$$
\left|G\left(t_{1}, s\right)-G\left(t_{2}, s\right)\right|<\frac{\epsilon}{2 M_{1}}
$$

for $t_{1}, t_{2} \in[0,1]$ with $\left|t_{1}-t_{2}\right|<\delta_{1}$ and $s \in[0,1]$. Therefore,

$$
\left|(A x)\left(t_{1}\right)-(A x)\left(t_{2}\right)\right| \leq \int_{0}^{1}\left|G\left(t_{1}, s\right)-G\left(t_{2}, s\right)\right| f(s, x(s)) d s<\epsilon, \quad x \in D,
$$

which means that $A(D)$ is equicontinuous. Noting that

$$
\left|\left(T_{\mu} x\right)\left(t_{1}\right)-\left(T_{\mu} x\right)\left(t_{2}\right)\right| \leq\left(M_{1} \int_{0}^{1} k(s) d s+\mu\right)\left|t_{1}-t_{2}\right|, \quad x \in D,
$$

we can show that $B(D)$ are equicontinuous. Applying the Arzelà-Ascoli theorem, we obtain that $A$ and $T_{\mu}$ are completely continuous. Moreover, $C_{(\lambda, \mu)}$ is completely continuous. This completes the proof.

\section{Existence and nonexistence results}

In this section, we apply Lemma 1.4 to establish the eigenvalue intervals for the existence of at least two, one, and no positive solutions for FEP (1) according to the range of the disturbance parameter $\mu$. We assume the following conditions:

(H1) $f(t, x)$ is nondecreasing in $x \in[0,+\infty)$ for fixed $t \in[0,1]$;

(H2) $g(x)$ is nondecreasing in $x \in[0,+\infty)$;

(H3) there exists $r_{0}>0$ such that $g\left(r_{0}\right) \int_{0}^{1} k(s) d s<r_{0}$;

(H4) $g_{\infty}:=\liminf _{x \rightarrow+\infty} \frac{g(x)}{x}>\frac{a+b}{\gamma_{0}\left(a \sigma_{1}+b\right) \int_{\sigma_{1}}^{\sigma_{2}} k(s) d s}$ and $\int_{\sigma_{1}}^{\sigma_{2}} k(s) d s>0$;

(H5) $f_{\infty}:=\lim \sup _{x \rightarrow+\infty} \min _{t \in\left[\sigma_{1}, \sigma_{2}\right]} \frac{f(t, x)}{x}=+\infty$.

In this section, we assume that $f(t, 0) \not \equiv 0, t \in[0,1]$.

Lemma 3.1 Suppose that $(\mathrm{H} 1)$ and $(\mathrm{H} 2)$ hold. If $x^{*} \in P$ is a fixed point of $C_{(\lambda, \mu)}$ and $\lambda+\mu \neq$ 0 , then $x^{*} \neq \theta$; moreover, $x^{*}$ is a positive solution of FEP (1).

Proof From (H1) and (H2) it is clear that

$$
x^{*}(t)=\left(C_{(\lambda, \mu)} x^{*}\right)(t) \geq \lambda \int_{0}^{1} G(t, s) f(s, 0) d s+\mu e(t),
$$

together with $\lambda+\mu \neq 0$ leads to $x^{*} \neq \theta$. It follows from Lemma 2.5 that $x^{*}$ is a positive solution of FEP (1). This completes the proof.

From Lemma 2.6 we have $C_{(\lambda, \mu)}(P) \subset K$. If (H1) and (H2) hold and $\lambda+\mu \neq 0$, then by Lemma 3.1, $x^{*} \in K$ is not a fixed point of $C_{(\lambda, \mu)}$, which implies that $x^{*}$ is not a positive solution of FEP (1). 
Lemma 3.2 Suppose that $(\mathrm{H} 1)$ and $(\mathrm{H} 2)$ hold.

(i) If there exist $\lambda_{0}, \mu_{0}>0$ such that $C_{\left(\lambda_{0}, \mu_{0}\right)}$ has a fixed point $x_{0} \in K$, then $C_{(\lambda, \mu)}$ has a fixed point $x \in K$ for any $0 \leq \lambda \leq \lambda_{0}, 0 \leq \mu \leq \mu_{0}, \lambda+\mu \neq 0$.

(ii) If there exist $\lambda_{0}^{\prime}, \mu_{0}^{\prime}>0$ such that $C_{\left(\lambda_{0}^{\prime}, \mu_{0}^{\prime}\right)}$ has no fixed points in $K$, then $C_{(\lambda, \mu)}$ has no fixed points in $K$ for $\lambda \geq \lambda_{0}^{\prime}, \mu \geq \mu_{0}^{\prime}$.

Proof From (H1) and (H2) it is easy to show that $C_{(\lambda, \mu)}: K \rightarrow K$ is an increasing operator. For any $0 \leq \lambda \leq \lambda_{0}$ and $0 \leq \mu \leq \mu_{0}$ with $\lambda+\mu \neq 0$, we have

$$
\left(C_{(\lambda, \mu)} x_{0}\right)(t) \leq\left(C_{\left(\lambda_{0}, \mu_{0}\right)} x_{0}\right)(t)=x_{0}(t), \quad t \in[0,1] .
$$

Set $w_{0}=x_{0}, w_{n}=C_{(\lambda, \mu)} w_{n-1}, n=1,2, \ldots$, then

$$
w_{0}(t) \geq w_{1}(t) \geq \cdots \geq w_{n}(t) \geq \cdots \geq \lambda \int_{0}^{1} G(t, s) f(s, 0) d s+\mu e(t) .
$$

By Lemma 2.6, $\left\{w_{n}\right\}$ converges to a fixed point $x$ of $C_{(\lambda, \mu)}$ in $K$.

It is evident that conclusion (i) implies conclusion (ii). This completes the proof.

Denote $f_{m}(x)=\max _{t \in[0,1]} f(t, x)$ for $x \geq 0$. If (H1) holds, then $f_{m}(x)>0$ for $x \geq 0$.

Theorem 3.3 Suppose that (H1), (H2), and (H3) hold. Then there exist $\lambda_{0}, \mu_{0}>0$ such that $C_{(\lambda, \mu)}$ has at least one fixed point $x \in K$ for $0 \leq \lambda \leq \lambda_{0}$ and $0 \leq \mu \leq \mu_{0}$ with $\lambda+\mu \neq 0$.

Proof Setting

$$
\lambda_{0}=\frac{\left(r_{0}-g\left(r_{0}\right) \int_{0}^{1} k(s) d s\right) \Gamma(\alpha+1)}{2 f_{m}\left(r_{0}\right)}, \quad \mu_{0}=\frac{r_{0}-g\left(r_{0}\right) \int_{0}^{1} k(s) d s}{2},
$$

and $w_{0}(t)=r_{0} e(t)$, we have

$$
\begin{aligned}
\left(C_{(\lambda, \mu)} w_{0}\right)(t) & \leq e(t)\left(\frac{\lambda_{0}}{\Gamma(\alpha)} \int_{0}^{1}(1-s)^{\alpha-1} f\left(s, r_{0}\right) d s+g\left(r_{0}\right) \int_{0}^{1} k(s) d s+\mu_{0}\right) \\
& \leq e(t)\left(\frac{\lambda_{0} f_{m}\left(r_{0}\right)}{\Gamma(\alpha+1)}+g\left(r_{0}\right) \int_{0}^{1} k(s) d s+\mu_{0}\right)=w_{0}(t), \quad t \in[0,1] .
\end{aligned}
$$

Let $w_{n}=C_{\left(\lambda_{0}, \mu_{0}\right)} w_{n-1}, n=1,2, \ldots$. Similarly to the proof of Lemma 3.2, we obtain that $\left\{w_{n}\right\}$ converges to a fixed point $x_{0}$ of $C_{\left(\lambda_{0}, \mu_{0}\right)}$ in $K$. Applying Lemma 3.2, the proof can be completed.

Theorem 3.4 Suppose that (H1), (H3), and (H4) hold. If $g(x)$ is strictly increasing in $x \in$ $[0,+\infty)$, then there exist $\mu^{*}>0$ and $\lambda^{*}:\left[0, \mu^{*}\right] \rightarrow[0,+\infty)$ satisfying

$$
\lambda^{*}(\mu)>0 \quad \text { as } 0 \leq \mu<\mu^{*}, \quad \lambda^{*}(\mu) \geq 0 \quad \text { as } \mu=\mu^{*} \text {, }
$$

such that

(i) FEP (1) has at least two positive solutions for $0 \leq \mu<\mu^{*}$ and $0<\lambda<\lambda^{*}(\mu)$; 
(ii) FEP (1) has at least one positive solution for $0 \leq \mu \leq \mu^{*}, \lambda=\lambda^{*}(\mu)$ or $\mu=\mu^{*}$,

$0 \leq \lambda \leq \lambda^{*}\left(\mu^{*}\right)$ or $0<\mu \leq \mu^{*}, \lambda=0 ;$

(iii) FEP (1) has no positive solutions for $0 \leq \mu \leq \mu^{*}, \lambda>\lambda^{*}(\mu)$ or $\mu>\mu^{*}, \lambda \geq 0$.

Proof We prove all statements by five steps.

Step 1. Set

$$
\Lambda=\left\{\mu \geq 0 \mid \exists \lambda>0, x_{\lambda} \in K \text { s.t., } C_{(\lambda, \mu)} x_{\lambda}=x_{\lambda}\right\}
$$

and

$$
\mu^{*}=\sup \Lambda
$$

By Theorem 3.3 we obtain that $\Lambda$ is nonempty and $0<\mu^{*} \leq+\infty$. If $\mu^{*}=+\infty$, then there exists an increasing sequence $\left\{\mu_{n}\right\}_{1}^{+\infty} \subset \Lambda$ such that $\lim _{n \rightarrow+\infty} \mu_{n}=+\infty$. By (12) there exist $\lambda_{n}>0$ and $x_{n} \in K$ such that $C_{\left(\lambda_{n}, \mu_{n}\right)} x_{n}=x_{n}$. There are two cases to be considered.

Case $1 .\left\{x_{n}\right\}_{1}^{+\infty}$ is bounded, that is, there exists a constant $M>0$ such that $\left\|x_{n}\right\| \leq M$ for $n=1,2, \ldots$. Then we have

$$
M \geq\left\|x_{n}\right\|=\left\|C_{\left(\lambda_{n}, \mu_{n}\right)} x_{n}\right\| \geq \mu_{n} \rightarrow+\infty
$$

which is a contradiction.

Case 2. $\left\{x_{n}\right\}_{1}^{+\infty}$ is unbounded, that is, there exists a subsequence of $\left\{x_{n}\right\}_{1}^{+\infty}$, still denoted by $\left\{x_{n}\right\}_{1}^{+\infty}$, such that $\lim _{n \rightarrow+\infty}\left\|x_{n}\right\|=+\infty$.

Choose $\epsilon>0$ such that $g_{\infty}-\epsilon>\frac{a+b}{\gamma_{0}\left(a \sigma_{1}+b\right) \int_{\sigma_{1}}^{\sigma_{2}} k(s) d s}$. By (H4) there exists $N_{1}>1$ such that $g(x) \geq\left(g_{\infty}-\epsilon\right) x$ for $x \geq N_{1}$. Choose $n_{1}$ such that $\left\|x_{n_{1}}\right\|>\frac{a+b}{\gamma_{0}\left(a \sigma_{1}+b\right)} N_{1}$. Then

$$
\min _{t \in\left[\sigma_{1}, \sigma_{2}\right]} x_{n_{1}}(t) \geq \frac{\gamma_{0}\left(a \sigma_{1}+b\right)}{a+b}\left\|x_{n_{1}}\right\| \geq N_{1} .
$$

Moreover,

$$
\begin{aligned}
\left\|x_{n_{1}}\right\| & =\left\|C_{\left(\lambda_{n_{1}}, \mu_{n_{1}}\right)} x_{n_{1}}\right\| \geq \int_{\sigma_{1}}^{\sigma_{2}} k(s) g\left(x_{n_{1}}(s)\right) d s \geq\left(g_{\infty}-\epsilon\right) \int_{\sigma_{1}}^{\sigma_{2}} k(s) x_{n_{1}}(s) d s \\
& \geq\left\|x_{n_{1}}\right\|\left(g_{\infty}-\epsilon\right) \frac{\gamma_{0}\left(a \sigma_{1}+b\right)}{a+b} \int_{\sigma_{1}}^{\sigma_{2}} k(s) d s>\left\|x_{n_{1}}\right\|,
\end{aligned}
$$

which is a contradiction. Consequently, $0<\mu^{*}<+\infty$; moreover,

$$
\left[0, \mu^{*}\right) \subset \Lambda
$$

Step 2. When $\mu=\mu^{*}$, we prove that there exist $\bar{\lambda} \geq 0$ and $\bar{x} \in K$ such that $C_{\left(\bar{\lambda}, \mu^{*}\right)} \bar{x}=\bar{x}$.

By (13) there exists an increasing sequence $\left\{\mu_{n}\right\}_{1}^{+\infty} \subset \Lambda$ such that $\lim _{n \rightarrow+\infty} \mu_{n}=\mu^{*}$. By (12) there exist $\lambda_{n}>0$ and $x_{n} \in K$ such that $C_{\left(\lambda_{n}, \mu_{n}\right)} x_{n}=x_{n}$. Arguing similarly to Case 2 , we can show that $\left\{x_{n}\right\}_{1}^{+\infty}$ is bounded, that is, there exists a constant $M>0$ such that $\left\|x_{n}\right\| \leq M$, $n=1,2, \ldots$. Arguing similarly to Case 1 , we obtain that $\left\{\lambda_{n}\right\}_{1}^{+\infty}$ is bounded and denote $\lambda^{\prime}=\sup \left\{\lambda_{n} \mid n=1,2, \ldots\right\}$. 
On the other hand, from

$$
\begin{aligned}
\left|x_{n}\left(t_{1}\right)-x_{n}\left(t_{2}\right)\right| \leq & \lambda^{\prime} \int_{0}^{1}\left|G\left(t_{1}, s\right)-G\left(t_{2}, s\right)\right| f(s, M) d s \\
& +\frac{a\left|t_{1}-t_{2}\right|}{a+b}\left(g(M) \int_{0}^{1} k(s) d s+\mu^{*}\right)
\end{aligned}
$$

it follows that $\left\{x_{n}\right\}_{1}^{+\infty}$ is equicontinuous. By the Arzelà-Ascoli theorem we conclude that $\left\{x_{n}\right\}_{1}^{+\infty}$ is relatively compact. Hence, there exist subsequences $\left\{\lambda_{n_{i}}\right\} \subset\left\{\lambda_{n}\right\}$ converging to $\bar{\lambda} \geq 0$ and $\left\{x_{n_{i}}\right\} \subset\left\{x_{n}\right\}$ converging to $\bar{x} \in K$. Since

$$
x_{n_{i}}(t)=\lambda_{n_{i}} \int_{0}^{1} G(t, s) f\left(s, x_{n_{i}}(s)\right) d s+e(t)\left(\int_{0}^{1} k(s) g\left(x_{n_{i}}(s)\right) d s+\mu_{n_{i}}\right),
$$

by taking the limit we have

$$
\bar{x}(t)=\bar{\lambda} \int_{0}^{1} G(t, s) f(s, \bar{x}(s)) d s+e(t)\left(\int_{0}^{1} k(s) g(\bar{x}(s)) d s+\mu^{*}\right),
$$

that is,

$$
C_{\left(\bar{\lambda}, \mu^{*}\right)} \bar{x}=\bar{x} \quad \text { and } \quad \bar{x} \in K
$$

Step 3. Let us define a function $\lambda^{*}:\left[0, \mu^{*}\right] \rightarrow[0,+\infty)$ satisfying (11). For any given $\mu \in$ $\left[0, \mu^{*}\right)$, set

$$
\Upsilon_{\mu}=\left\{\lambda>0 \mid \exists x_{\lambda} \in K \text { s.t., } C_{(\lambda, \mu)} x_{\lambda}=x_{\lambda}\right\}
$$

and

$$
\lambda^{*}(\mu)=\sup \Upsilon_{\mu} .
$$

By a similar argument as for (14) we can obtain that $0<\lambda^{*}(\mu)<+\infty$.

Next, we show that $\lambda^{*}(\mu) \in \Upsilon_{\mu}$. By (17) there exists an increasing sequence $\left\{\lambda_{n}\right\}_{1}^{+\infty} \subset$ $\Upsilon_{\mu}$ such that $\lim _{n \rightarrow+\infty} \lambda_{n}=\lambda^{*}(\mu)$. By (16) there exist $x_{\lambda_{n}} \in K$ such that $C_{\left(\lambda_{n}, \mu\right)} x_{\lambda_{n}}=x_{\lambda_{n}}$. Similarly, we can obtain that there exists a subsequence $\left\{x_{\lambda_{n_{i}}}\right\} \subset\left\{x_{\lambda_{n}}\right\}$ converging to $x^{*} \in K$ and

$$
x^{*}(t)=\left(C_{(\lambda *(\mu), \mu)} x^{*}\right)(t) .
$$

From (16) we have $\lambda^{*}(\mu) \in \Upsilon_{\mu}$. Lemma 3.2 implies

$$
\left(0, \lambda^{*}(\mu)\right]=\Upsilon_{\mu}, \quad 0 \leq \mu<\mu^{*} .
$$

When $\mu=\mu^{*}$, set

$$
\Upsilon_{\mu^{*}}=\left\{\lambda \geq 0 \mid \exists x_{\lambda} \in K \text { s.t., } C_{\left(\lambda, \mu^{*}\right)} x_{\lambda}=x_{\lambda}\right\},
$$




$$
\lambda^{*}\left(\mu^{*}\right)=\sup \Upsilon_{\mu^{*}}
$$

Then, we can show that $0 \leq \lambda^{*}\left(\mu^{*}\right)<+\infty$ and $\lambda^{*}\left(\mu^{*}\right) \in \Upsilon_{\mu^{*}}$, that is,

$$
\left[0, \lambda^{*}\left(\mu^{*}\right)\right]=\Upsilon_{\mu^{*}}
$$

By the preceding discussion we obtain that $\mu^{*}>0$ and that the function $\lambda^{*}:\left[0, \mu^{*}\right] \rightarrow$ $[0,+\infty)$ defined by (17) and (20) satisfies (11)

Step 4. It is evident by (14), (15), (18), (21), and the discussion of Step 2, together with Lemma 3.2. that conclusions (ii) and (iii) hold.

Step 5. We prove conclusion (i), that is, that FEP (1) has at least two positive solutions for $0 \leq \mu<\mu^{*}$ and $0<\lambda<\lambda^{*}(\mu)$.

Given $\mu \in\left[0, \mu^{*}\right)$ and $\lambda \in\left(0, \lambda^{*}(\mu)\right)$, let $\bar{x}^{*} \in K$ be a fixed point of $C_{\left(\lambda^{*}(\mu), \mu\right)}$. Then

$$
\left(C_{(\lambda, \mu)} \bar{x}^{*}\right)(t) \leq \lambda^{*}(\mu) \int_{0}^{1} G(t, s) f\left(s, \bar{x}^{*}(s)\right) d s+\left(\int_{0}^{1} k(s) g\left(\bar{x}^{*}(s)\right) d s+\mu\right) e(t)=\bar{x}^{*}(t) .
$$

Similarly to the proof of Theorem 3.3, we obtain that $C_{(\lambda, \mu)}$ has at least one fixed point $x_{1}^{*} \in K$ and $x_{1}^{*}(t) \leq \bar{x}^{*}(t)$ for $t \in[0,1]$. Let

$$
\Omega_{1}=\left\{x \in K \mid x(t)<\bar{x}^{*}(t), t \in[0,1]\right\} .
$$

Then $\Omega_{1}$ is a nonempty open bounded set in $K$. Let us prove that

$$
x_{1}^{*} \in \Omega_{1} \text {. }
$$

Indeed, noting that $x_{1}^{*} \in K$ and $x_{1}^{*}(t) \leq \bar{x}^{*}(t)$ for $t \in[0,1]$, we only need to prove that $x_{1}^{*}(t)<$ $\bar{x}^{*}(t)$ for $t \in[0,1]$. If $x_{1}^{*}(t)=\bar{x}^{*}(t)$ for $t \in[0,1]$, then

$$
\left.0=\left(\lambda^{*}(\mu)-\lambda\right) \int_{0}^{1} G(t, s) f\left(s, x_{1}^{*}(s)\right) d s \geq\left(\lambda^{*}(\mu)-\lambda\right) \int_{0}^{1} G(t, s) f(s, 0)\right) d s>0,
$$

which is a contradiction. So $x_{1}^{*} \neq \bar{x}^{*}$. There are two cases to be considered.

Case 1 . There exists $t_{0} \in[0,1)$ such that $x_{1}^{*}\left(t_{0}\right)=\bar{x}^{*}\left(t_{0}\right)$. This means that

$$
\begin{gathered}
\int_{0}^{1} G\left(t_{0}, s\right)\left(\lambda^{*}(\mu) f\left(s, \bar{x}^{*}(s)\right)-\lambda f\left(s, x_{1}^{*}(s)\right)\right) d s \\
=e\left(t_{0}\right) \int_{0}^{1} k(s)\left(g\left(x_{1}^{*}(s)\right)-g\left(\bar{x}^{*}(s)\right)\right) d s .
\end{gathered}
$$

It is easy to check that

the left side of $(23) \geq\left(\lambda^{*}(\mu)-\lambda\right) \int_{0}^{1} G\left(t_{0}, s\right) f\left(s, \bar{x}^{*}(s)\right) d s>0$, and

the right side of $(23)=e\left(t_{0}\right) \int_{0}^{1} k(s)\left(g\left(x_{1}^{*}(s)\right)-g\left(\bar{x}^{*}(s)\right)\right) d s \leq 0$, which is a contradiction.

Case 2. If $x_{1}^{*}(t)<\bar{x}^{*}(t)$ for $t \in[0,1)$ and $x_{1}^{*}(1)=\bar{x}^{*}(1)$, then

$$
\int_{0}^{1} k(s)\left(g\left(x_{1}^{*}(s)\right)-g\left(\bar{x}^{*}(s)\right)\right) d s=0,
$$


which implies that $g\left(x_{1}^{*}(t)\right)=g\left(\bar{x}^{*}(t)\right)$ for $t \in[0,1)$. This contradicts the fact that $g(x)$ is strictly increasing in $x$. So (22) holds.

Now, we find the second positive solution of FEP (1). Set

$$
\begin{aligned}
& F(t, x(t))= \begin{cases}f\left(t, \bar{x}^{*}(t)\right), & x(t)>x^{*}(t), \\
f(t, x(t)), & 0 \leq x(t) \leq x^{*}(t),\end{cases} \\
& G(x(t))= \begin{cases}g\left(\bar{x}^{*}(t)\right), & x(t)>x^{*}(t), \\
g(x(t)), & 0 \leq x(t) \leq x^{*}(t) .\end{cases}
\end{aligned}
$$

Consider the following fractional boundary value problem:

$$
\left\{\begin{array}{l}
{ }^{C} D_{0^{+}}^{\alpha} x(t)+\lambda F(t, x(t))=0, \quad 0<t<1, \\
a x(0)-b x^{\prime}(0)=0, \\
x(1)=\int_{0}^{1} k(s) G(x(s)) d s+\mu .
\end{array}\right.
$$

Lemmas 2.4 and 2.5 imply that $x$ is a positive solution of FEP (24) if and only if $x$ is a positive fixed point of the operator $\bar{C}_{(\lambda, \mu)}$ defined by

$$
\left(\bar{C}_{(\lambda, \mu)} x\right)(t)=\lambda \int_{0}^{1} G(t, s) F(s, x(s)) d s+\left(\int_{0}^{1} k(s) G(x(s)) d s+\mu\right) e(t), \quad x \in K .
$$

Since $F$ and $G$ are bounded, there exists $R>\left\|\bar{x}^{*}\right\|$ such that

$$
\left\|\bar{C}_{(\lambda, \mu)} x\right\|<R, \quad x \in K
$$

Set

$$
K_{R}=\{x \in K \mid\|x\|<R\} .
$$

Then $\bar{\Omega}_{1} \subset K_{R}$. Similarly to the proof of Lemma 2.6 , we can show that $\bar{C}_{(\lambda, \mu)}: \bar{K}_{R} \rightarrow K$ is a completely continuous operator. By (26) and Lemma 1.4 we have

$$
i\left(\bar{C}_{(\lambda, \mu)}, K_{R}, K\right)=1 \text {. }
$$

We assert that $\bar{C}_{(\lambda, \mu)}$ has no fixed points in $\bar{K}_{R} \backslash \Omega_{1}$. Indeed, if $y^{*}(t) \in K$ is a fixed point of $\bar{C}_{(\lambda, \mu)}$, then by the definition of $F$ and $G$ we obtain

$$
\begin{aligned}
y^{*}(t) & =\lambda \int_{0}^{1} G(t, s) F\left(s, y^{*}(s)\right) d s+\left(\int_{0}^{1} k(s) G\left(y^{*}(s)\right) d s+\mu\right) e(t) \\
& \leq \lambda^{*}(\mu) \int_{0}^{1} G(t, s) f\left(s, \bar{x}^{*}(s)\right) d s+\left(\int_{0}^{1} k(s) g\left(\bar{x}^{*}(s)\right) d s+\mu\right) e(t)=\bar{x}^{*}(t),
\end{aligned}
$$

which implies that $y^{*} \in \bar{\Omega}_{1}$. Note that $\bar{C}_{(\lambda, \mu)} x=C_{(\lambda, \mu)} x$ for $x \in \bar{\Omega}_{1}$, and from the proof of (22) we have $y^{*} \in \Omega_{1}$. Hence, by the excision property of the fixed point index we have

$$
i\left(C_{(\lambda, \mu)}, \Omega_{1}, K\right)=i\left(\bar{C}_{(\lambda, \mu)}, \Omega_{1}, K\right)=i\left(\bar{C}_{(\lambda, \mu)}, K_{R}, K\right)=1 .
$$


Choose $\epsilon>0$ such that $g_{\infty}-\epsilon>\frac{a+b}{\left(a \sigma_{1}+b\right) \gamma_{0} \int_{\sigma_{1}}^{\sigma_{2}} k(s) d s}$. By (H4) there exists $R^{\prime}>R$ such that $g(x) \geq\left(g_{\infty}-\epsilon\right) x$ for $x \geq R^{\prime}$. Let

$$
\Omega_{2}=\left\{x \in K \mid\|x\|<\frac{a+b}{\gamma_{0}\left(a \sigma_{1}+b\right)} R^{\prime}\right\} .
$$

Noting that $R<R^{\prime}<\frac{a+b}{\gamma_{0}\left(a \sigma_{1}+b\right)} R^{\prime}$, we have $\bar{\Omega}_{1} \subset \Omega_{2}$.

For $x \in \partial \Omega_{2}$, noting that $\min _{t \in\left[\sigma_{1}, \sigma_{2}\right]} x(t) \geq \gamma_{0} e\left(\sigma_{1}\right)\|x\| \geq R^{\prime}$, we have

$$
\begin{aligned}
\left(C_{(\lambda, \mu)} x\right)(t) & \geq e(t) \int_{\sigma_{1}}^{\sigma_{2}} k(s) g(x(s)) d s \geq\left(g_{\infty}-\epsilon\right) e(t) \int_{\sigma_{1}}^{\sigma_{2}} k(s) x(s) d s \\
& \geq \frac{\left(a \sigma_{1}+b\right) \gamma_{0}\left(g_{\infty}-\epsilon\right) e(t)\|x\|}{a+b} \int_{\sigma_{1}}^{\sigma_{2}} k(s) d s,
\end{aligned}
$$

which means that

$$
\left\|C_{(\lambda, \mu)} x\right\| \geq \frac{\left(a \sigma_{1}+b\right)\left(g_{\infty}-\epsilon\right) \gamma_{0}\|x\|}{a+b} \int_{\sigma_{1}}^{\sigma_{2}} k(s) d s>\|x\| .
$$

By Lemma 1.4 we have

$$
i\left(C_{(\lambda, \mu)}, \Omega_{2}, K\right)=0 .
$$

According to the additivity of the fixed point index, we get

$$
i\left(C_{(\lambda, \mu)}, \Omega_{2} \backslash \bar{\Omega}_{1}, K\right)=i\left(C_{(\lambda, \mu)}, \Omega_{2}, K\right)-i\left(C_{(\lambda, \mu)}, \Omega_{1}, K\right)=-1,
$$

which implies that $C_{(\lambda, \mu)}$ has at least one fixed point $x_{2}^{*}$ in $\bar{\Omega}_{2} \backslash \Omega_{1}$. Therefore, FEP (1) has another positive solution $x_{2}^{*}$. The proof is complete.

Remark 3.5 We can give an estimate for the critical value $\mu^{*}$ in Theorem 3.4. In fact, letting $w_{0}=r_{0} e$, from the proof of Theorem 3.3 we have $C_{(\lambda, \mu)} w_{0}(t) \leq w_{0}(t)$, provided that

$$
\frac{\lambda f_{m}\left(r_{0}\right)}{\Gamma(\alpha+1)}+g\left(r_{0}\right) \int_{0}^{1} k(s) d s+\mu \leq r_{0} .
$$

Hence, for $0 \leq \mu<r_{0}-g\left(r_{0}\right) \int_{0}^{1} k(s) d s$, there exist

$$
\lambda=\frac{\left(r_{0}-g\left(r_{0}\right) \int_{0}^{1} k(s) d s-\mu\right) \Gamma(\alpha+1)}{f_{m}\left(r_{0}\right)} \geq 0
$$

and $x_{\lambda} \in K$ such that $C_{(\lambda, \mu)} x_{\lambda}=x_{\lambda}$ and $\lambda+\mu>0$. That is, $\left[0, r_{0}-g\left(r_{0}\right) \int_{0}^{1} k(s) d s\right) \subset \Lambda$, which means that

$$
\mu^{*} \geq r_{0}-g\left(r_{0}\right) \int_{0}^{1} k(s) d s .
$$

In particular, if $0 \leq \mu \leq r_{0}-g\left(r_{0}\right) \int_{0}^{1} k(s) d s$, then

$$
\lambda^{*}(\mu) \geq \frac{\left(r_{0}-g\left(r_{0}\right) \int_{0}^{1} k(s) d s-\mu\right) \Gamma(\alpha+1)}{f_{m}\left(r_{0}\right)} .
$$


Similarly to the proof of Theorem 3.4, we obtain the following result.

Theorem 3.6 Suppose that (H1), (H2), (H3) and (H5) hold. Then, there exist $\lambda^{*}>0$ and $\mu^{*}:\left(0, \lambda^{*}\right] \rightarrow[0,+\infty)$ satisfying

$$
\mu^{*}(\lambda)>0 \quad \text { for } 0 \leq \lambda<\lambda^{*}, \quad \mu^{*}(\lambda) \geq 0 \quad \text { for } \lambda=\lambda^{*},
$$

such that:

(i) FEP (1) has at least two positive solutions for $0<\lambda<\lambda^{*}$ and $0 \leq \mu<\mu^{*}(\lambda)$;

(ii) FEP (1) has at least one positive solution for $0 \leq \lambda<\lambda^{*}, \mu=\mu^{*}(\lambda)$, or $\lambda=\lambda^{*}$, $0 \leq \mu \leq \mu^{*}(\lambda)$, or $0<\lambda \leq \lambda^{*}, \mu=0$;

(iii) FEP (1) has no positive solutions for $0 \leq \lambda \leq \lambda^{*}, \mu>\mu^{*}(\lambda)$ or $\lambda>\lambda^{*}, \mu \geq 0$.

Remark 3.7 Feng and Zhang [38] studied the existence of solutions for the operator equation

$$
T x=x, \quad x \in P_{u_{0}},
$$

where $X_{+}$is the positive cone of an ordered Banach space $X$, and

$$
P_{u_{0}}=\left\{x \in X_{+} \mid x \geq\|x\| u_{0}\right\}, \quad u_{0} \in X_{+},\left\|u_{0}\right\| \leq 1
$$

is a subcone of $X_{+}$, and obtained some meaningful conclusions, which can be applied to an abundance of concrete problems. Note that the cone $K$ used in this paper is not a particular case of the cone $P_{u_{0}}$; our results in this paper differ from the applications of the abstract results in [38].

\section{Uniqueness and dependence on parameter}

In this section, we apply Lemmas 1.5 and 1.6 and the cone theory to further study the maximum eigenvalue interval for the existence of the unique positive solution for FEP (1) and the dependence of such a positive solution on the parameter $\lambda$ for given $\mu>0$. We need the following hypotheses:

(H6) for any $r \in(0,1)$ and $x \in[0,+\infty), f(t, r x) \geq r f(t, x)$ for $t \in[0,1]$;

(H7) for any $r \in(0,1)$ and $x \in[0,+\infty), g(r x) \geq r g(x)$;

(H8) $\lim _{x \rightarrow+\infty} g(x)<+\infty$;

(H9) there exists a constant $\kappa \in(0,1)$ such that

$$
f(t, r x) \geq r^{k} f(t, x), \quad \forall t \in[0,1], r \in(0,1), x \in[0, \infty) .
$$

Let $e(t)=\frac{a t+b}{a+b}$. Define $P_{e}$ and $P_{e}^{*}$ as in Section 1 and the operators $A$ and $T_{\mu}$ as in Section 2 .

Lemma 4.1 Assume that (H1) and (H2) hold. Then

(i) $A: P \rightarrow P$ is an increasing operator, and $A(P) \subset P_{e}^{*}$;

(ii) $T_{\mu}: P \rightarrow P$ is an increasing operator, and $T_{\mu}(P) \subset P_{e}$ for $\mu>0$;

moreover, $C_{(\lambda, \mu)}: P \rightarrow P_{e}$ is increasing for $\lambda \geq 0$ and $\mu>0$. 
Proof From Lemma 2.2 we have

$$
\begin{aligned}
& 0 \leq(A x)(t) \leq e(t) \int_{0}^{1} \frac{(1-s)^{\alpha-1}}{\Gamma(\alpha)} f(s, x(s)) d s, \quad \forall x \in P, \\
& \mu e(t) \leq\left(T_{\mu} x\right)(t) \leq e(t)\left(\int_{0}^{1} k(s) g(x(s)) d s+\mu\right), \quad \forall x \in P, \mu>0 .
\end{aligned}
$$

This, together with (H1) and (H2), completes the proof.

Lemma 4.2 Assume that (H7) and (H8) hold. Then for given $\mu>0$, there exists $\tau \in(0,1)$ such that

$$
T_{\mu}(r x) \geq r^{\tau} T_{\mu} x, \quad x \in P_{e}, r \in(0,1) .
$$

Proof It is easy to show from (H8) that the function $g$ is bounded, that is, there exists $M>0$ such that

$$
g(x) \leq M, \quad x \in[0,+\infty)
$$

Let $\tau=\frac{M \int_{0}^{1} k(s) d s}{\mu+M \int_{0}^{1} k(s) d s}$; then $0<\tau<1$. Set

$$
h(r)=\frac{1-r^{\tau}}{r^{\tau}-r}, \quad r \in(0,1),
$$

By straightforward calculations we get that $\frac{d h(r)}{d r}<0$ for $r \in(0,1)$. This means that

$$
\frac{1-r^{\tau}}{r^{\tau}-r}>\lim _{r \rightarrow 1-1-r^{\tau}-r} \frac{1-r^{\tau}}{1-\tau} \geq \frac{\int_{0}^{1} k(s) g(x(s)) d s}{\mu}, \quad r \in(0,1), x \in P_{e} .
$$

that is,

$$
\mu+r \int_{0}^{1} k(s) g(x(s)) d s \geq r^{\tau}\left(\mu+\int_{0}^{1} k(s) g(x(s)) d s\right), \quad r \in(0,1), x \in P_{e} .
$$

From (H7) we have

$$
T_{\mu}(r x)(t) \geq e(t)\left(\mu+r \int_{0}^{1} k(s) g(x(s)) d s\right) \geq r^{\tau}\left(T_{\mu} x\right)(t), \quad r \in(0,1), x \in P_{e} .
$$

This completes the proof.

Lemma 4.3 Assume that (H1), (H2), (H6), (H7), and (H8) hold. Then for any $r \in(0,1)$ and $x \in P_{e}$, there exists $\eta(r, x)>0$ such that

$$
C_{(\lambda, \mu)}(r x) \geq r(1+\eta(r, x)) C_{(\lambda, \mu)} x
$$

for $\lambda \geq 0$ and $\mu>0$. 
Proof Take

$$
\eta(r, x)=\frac{\left(r^{\tau-1}-1\right) \mu}{\lambda \int_{0}^{1} \frac{(1-s)^{\alpha-1}}{\Gamma(\alpha)} f(s,\|x\|) d s+M \int_{0}^{1} k(s) d s+\mu}, \quad r \in(0,1), x \in P_{e},
$$

where $M$ is given by (28); then $\eta(r, x)>0$. Then, from (H1), (H2), (H6), and Lemma 4.2 we have

$$
\begin{aligned}
C_{(\lambda, \mu)}(r x)(t) & \geq r \lambda A x(t)+r^{\tau} T_{\mu} x(t) \geq r C_{(\lambda, \mu)} x(t)+\left(r^{\tau}-r\right) \mu e(t) \\
& \geq r(1+\eta(r, x)) C_{(\lambda, \mu)} x(t), \quad r \in(0,1), x \in P_{e} .
\end{aligned}
$$

This completes the proof.

Remark 4.4 By Lemma 4.1 we have $C_{(\lambda, \mu)}(P) \subset P_{e}$. This means that if $x$ is not a fixed point of $C_{(\lambda, \mu)}$ in $P_{e}$, then $x$ is not a fixed point of $C_{(\lambda, \mu)}$ in $P$. So, in this section, we study the existence and nonexistence of the unique fixed point of $C_{(\lambda, \mu)}$ in $P_{e}$.

Theorem 4.5 Assume that (H1), (H2), (H6), (H7), and (H8) hold. Then for any given $\mu>0$, there exists $\lambda^{*}(\mu)>0$ such that FEP (1) has a unique positive solution $x_{\lambda}$ for $\lambda \in\left[0, \lambda^{*}(\mu)\right)$ and has no positive solution for $\lambda \geq \lambda^{*}(\mu)$. Furthermore, such a solution $x_{\lambda}$ satisfies the following properties:

(i) for any $u_{0} \in P$, setting $u_{n}(t)=\lambda \int_{0}^{1} G(t, s) f\left(s, u_{n-1}(s)\right) d s+e(t)\left(\int_{0}^{1} k(s) g\left(u_{n-1}(s)\right) d s+\right.$ $\mu), n=1,2, \ldots$, we have $\lim _{n \rightarrow \infty}\left\|u_{n}-x_{\lambda}\right\|=0$;

(ii) $x_{\lambda}$ is increasing in $\lambda$ for $\lambda \in\left[0, \lambda^{*}(\mu)\right)$;

(iii) $x_{\lambda}$ is continuous with respect to $\lambda$ for $\lambda \in\left[0, \lambda^{*}(\mu)\right)$.

Proof Given $\mu>0$, Lemma 4.1 and (H6) imply that $A$ satisfies condition (G1). It is easy to see by Lemmas 4.1 and 4.2 that $T_{\mu}$ satisfies condition (G2). Consequently, Theorem 4.5 follows from Lemma 1.5. The proof is complete.

Now, for given $\mu>0$, we estimate the critical value $\lambda^{*}(\mu)$ in Theorem 4.5. If (H1) and (H6) hold, then

$$
\frac{f(t, x)}{x} \leq f(t, 1) \leq \max _{t \in[0,1]} f(t, 1), \quad x>1, t \in[0,1] .
$$

Moreover, $F_{\infty}:=\lim \sup _{x \rightarrow+\infty} \max _{t \in[0,1]} \frac{f(t, x)}{x} \in[0,+\infty)$.

Theorem 4.6 Assume that (H1), (H2), (H6), (H7), and (H8) hold. Then for any $\mu>0$,

$$
\lambda^{*}(\mu) \begin{cases}\geq \frac{\Gamma(\alpha+1)}{2 F_{\infty}}, & 0<F_{\infty}<+\infty, \\ =+\infty, & F_{\infty}=0 .\end{cases}
$$

Proof Given $\mu>0$, Lemma 4.3, together with (29), implies that all the conditions in Lemma 1.6 are satisfied. For $\lambda \geq 0$, according to Lemma 1.6, $C_{(\lambda, \mu)}$ has a unique fixed point in $P_{e}$ if and only if there exist $u_{\lambda}, v_{\lambda} \in P_{e}$ such that

$$
u_{\lambda} \leq C_{(\lambda, \mu)} u_{\lambda} \leq C_{(\lambda, \mu)} v_{\lambda} \leq v_{\lambda}
$$


Take $u_{\lambda}=C_{(\lambda, \mu)} \theta$, where $\theta(t) \equiv 0$; then $u_{\lambda} \in P_{e}$. If there exists $v_{\lambda} \in P_{e}$ such that $C_{(\lambda, \mu)} v_{\lambda} \leq$ $v_{\lambda}$, by the increasing property of $C_{(\lambda, \mu)}$ and $\theta<v_{\lambda}$ we have

$$
C_{(\lambda, \mu)} \theta=u_{\lambda} \leq C_{(\lambda, \mu)} u_{\lambda} \leq C_{(\lambda, \mu)} v_{\lambda} \leq v_{\lambda}
$$

Therefore, $C_{(\lambda, \mu)}$ has a unique fixed point in $P_{e}$ if and only if there exists $v_{\lambda} \in P_{e}$ such that $C_{(\lambda, \mu)} v_{\lambda} \leq v_{\lambda}$. Set

$$
\Delta=\left\{\lambda \geq 0 \mid \text { there exists } v_{\lambda} \in P_{e} \text { such that } C_{(\lambda, \mu)} v_{\lambda} \leq v_{\lambda}\right\}
$$

Then $C_{(\lambda, \mu)}$ has a unique fixed point in $P_{e}$ if and only if $\lambda \in \Delta$. Moreover, by Theorem 4.5 it is easy to see that

$$
\lambda^{*}(\mu)=\sup \Delta \quad \text { and } \quad \lambda^{*}(\mu) \notin \Delta .
$$

On the other hand, for any $\epsilon>0$, by Lemma 4.2 and the definition of $F_{\infty}$ there exists $r_{0} \in(0,1)$ such that

$$
r_{0}^{1-\tau} e \leq T_{\mu} e \leq \frac{1}{2 r_{0}^{1-\tau}} e \quad \text { and } \quad f\left(t, \frac{1}{r}\right) \leq \frac{1}{r}\left(F_{\infty}+\epsilon\right), \quad r \leq r_{0}, t \in[0,1]
$$

Set $w(t)=\frac{1}{r_{0}} e(t)$. Then

$$
\begin{aligned}
& (A w)(t) \leq \frac{e(t)}{\Gamma(\alpha)} \int_{0}^{1}(1-s)^{\alpha-1} f\left(s, \frac{1}{r_{0}}\right) d s \leq \frac{e(t)\left(F_{\infty}+\epsilon\right)}{r_{0} \Gamma(\alpha+1)} \leq \frac{\left(F_{\infty}+\epsilon\right)}{\Gamma(\alpha+1)} w(t), \\
& \left(T_{\mu} w\right)(t) \leq \frac{1}{r_{0}^{\tau}}\left(T_{\mu} e\right)(t) \leq \frac{1}{2 r_{0}^{\tau} r_{0}^{1-\tau}} e(t)=\frac{1}{2} w(t) .
\end{aligned}
$$

Taking $\lambda_{\epsilon}=\frac{\Gamma(\alpha+1)}{2\left(F_{\infty}+\epsilon\right)}$, we have

$$
\left(C_{\left(\lambda_{\epsilon}, \mu\right)} w\right)(t)=\lambda_{\epsilon}(A w)(t)+\left(T_{\mu} w\right)(t) \leq \frac{\left(F_{\infty}+\epsilon\right) \lambda_{\epsilon}}{\Gamma(\alpha+1)} w(t)+\frac{1}{2} w(t) \leq w(t) .
$$

Therefore, from the definition of $\Delta$ we obtain that $\lambda_{\epsilon}=\frac{\Gamma(\alpha+1)}{2\left(F_{\infty}+\epsilon\right)} \in \Delta$, that is, $\lambda^{*}(\mu)>\frac{\Gamma(\alpha+1)}{2\left(F_{\infty}+\epsilon\right)}$, which implies that (30) holds. This completes the proof.

Corollary 4.7 Assume that (H1), (H2), (H7), (H8), and (H9) hold. Then for given $\mu>0$, FEP (1) has a unique positive solution $x_{\lambda}$ for $\lambda \in[0,+\infty)$. Furthermore, such a solution $x_{\lambda}$ satisfies the following properties:

(i) for any $u_{0} \in P$, setting $u_{n}=\lambda A u_{n-1}+T_{\mu} u_{n-1}(n=1,2, \ldots)$, we have $\lim _{n \rightarrow \infty}\left\|u_{n}-x_{\lambda}\right\|=0$

(ii) $x_{\lambda}$ is nondecreasing in $\lambda$ for $\lambda \in[0,+\infty)$;

(iii) $x_{\lambda}$ is continuous with respect to $\lambda$ for $\lambda \in[0,+\infty)$;

(iv) iff $(t, 1) \not \equiv 0$, then $\lim _{\lambda \rightarrow 0+}\left\|x_{\lambda}-x_{\mu}\right\|=0$ and $\lim _{\lambda \rightarrow+\infty}\left\|x_{\lambda}\right\|=+\infty$, where $x_{\mu}$ is the unique fixed point of $T_{\mu}$ in $P_{e}$. 
Proof It is clear that (H9) implies (H6) and

$$
F_{\infty}=\limsup _{x \rightarrow+\infty} \max _{t \in[0,1]} \frac{f(t, x)}{x}=0 .
$$

Applying Theorem 4.5 and Theorem 4.6, we complete the proofs of the all conclusions except (iv).

Next, we prove (iv). It is obvious from conclusion (iii) that $\lim _{\lambda \rightarrow 0+}\left\|x_{\lambda}-x_{\mu}\right\|=0$. Noting that $x_{\lambda}(t)=\left(C_{(\lambda, \mu)} x_{\lambda}\right)(t) \geq \mu e(t)$, from $(\mathrm{H} 1)$ and $(\mathrm{H} 9)$ we have

$$
x_{\lambda}(t) \geq \lambda \int_{0}^{1} G(t, s) f(s, \mu e(s)) d s \geq \lambda \min \{\mu, 1\} \int_{0}^{1} G(t, s) e(s) f(s, 1) d s .
$$

Hence,

$$
\left\|x_{\lambda}\right\| \geq \lambda \min \{\mu, 1\} \max _{t \in[0,1]} \int_{0}^{1} G(t, s) e(s) f(s, 1) d s,
$$

which means that $\lim _{\lambda \rightarrow+\infty}\left\|x_{\lambda}\right\|=+\infty$. This ends the proof.

When $g(x)$ is a constant function $c(\geq 0)$, it is evident that $g$ satisfies (H2), (H7), and (H8). For given $\mu>0, T_{\mu} x(t)=\left(c \int_{0}^{1} k(s) d s+\mu\right) e(t):=x_{\mu}(t)$. We can obtain the following results.

Corollary 4.8 Assume that $(\mathrm{H} 1)$ and $(\mathrm{H} 6)$ hold. If $F_{\infty}>0$, then, for given $\mu>0$ :

(i) there exists $\lambda^{*}(\mu) \geq \frac{\Gamma(\alpha+1)}{2 F_{\infty}}$ such that FEP (1) with $g \equiv$ c has a unique positive solution $x_{\lambda}$ for $\lambda \in\left[0, \lambda^{*}(\mu)\right)$ and has no solution for $\lambda \geq \lambda^{*}(\mu)$. Moreover, for any $u_{0} \in P$, setting $u_{n}=x_{\mu}+\lambda A u_{n-1}(n=1,2, \ldots)$, we have $\lim _{n \rightarrow \infty}\left\|u_{n}-x_{\lambda}\right\|=0$;

(ii) $x_{\lambda}$ is nondecreasing in $\lambda$ for $\lambda \in\left[0, \lambda^{*}(\mu)\right)$;

(iii) $x_{\lambda}$ is continuous with respect to $\lambda$ for $\lambda \in\left[0, \lambda^{*}(\mu)\right)$; moreover, $\lim _{\lambda \rightarrow 0+}\left\|x_{\lambda}-x_{\mu}\right\|=0$.

Corollary 4.9 Assume that $(\mathrm{H} 1)$ and $(\mathrm{H} 6)$ hold. If $F_{\infty}=0$, then, for given $\mu>0$ :

(i) FEP (1) with $g \equiv c$ has a unique positive solution $x_{\lambda}$ for any $\lambda \in[0,+\infty)$; moreover, for any $u_{0} \in P$, setting $u_{n}=x_{\mu}+\lambda A u_{n-1}(n=1,2, \ldots)$, we have $\lim _{n \rightarrow \infty}\left\|u_{n}-x_{\lambda}\right\|=0$;

(ii) $x_{\lambda}$ is nondecreasing in $\lambda$ for $\lambda \in[0,+\infty)$;

(iii) $x_{\lambda}$ is continuous with respect to $\lambda$ for $\lambda \in[0,+\infty)$;

(iv) $\lim _{\lambda \rightarrow 0+}\left\|x_{\lambda}-x_{\mu}\right\|=0$ and $\lim _{\lambda \rightarrow+\infty}\left\|x_{\lambda}\right\|=+\infty$.

Corollary 4.10 Assume that (H1) and (H9) hold. Then conclusions (i), (ii), (iii), and (iv) in Corollary 4.9 hold.

Remark 4.11 In the particular case of $\alpha=2$, FEP (1) can be regarded as the following second-order boundary value problem:

$$
\left\{\begin{array}{l}
x^{\prime \prime}(t)+\lambda f(t, x(t))=0, \quad 0<t<1, \\
a x(0)-b x^{\prime}(0)=0, \\
x(1)=\int_{0}^{1} k(s) g(x(s)) d s+\mu,
\end{array}\right.
$$

and all the results in this paper still hold. However, our research does not contain the case of FEP (1) with $\alpha=1$. Zhang and Cheng [39] studied the existence and nonexistence of 
positive periodic solutions for the first-order coupled functional differential system depending on two parameters

$$
\left\{\begin{array}{l}
x^{\prime}(t)=-a_{1}(t) x(t)+\lambda k_{1}(s) f_{1}\left(x\left(t-\tau_{1}(t)\right), y\left(t-\sigma_{1}(t)\right)\right), \\
y^{\prime}(t)=-a_{2}(t) y(t)+\mu k_{2}(s) f_{2}\left(x\left(t-\tau_{2}(t)\right), y\left(t-\sigma_{2}(t)\right)\right),
\end{array}\right.
$$

where $a_{i}, k_{i}, \tau_{i}, \sigma_{i}$ are continuous $\omega(>0)$-periodic functions, $f_{i}, k_{i}$ are positive continuous functions, and $\int_{0}^{\omega} a_{i}(t) d t>0, i=1,2$, and obtain some interesting results. In comparison with [39], we are concerned with the eigenvalue problem of fractional $\alpha(1<\alpha \leq 2)$ order differential equations (1) with a disturbance parameter in the boundary conditions, which is different from the first-order differential system (31) in [39]. In addition, we study not only the existence and nonexistence of positive solutions, but also the existence and uniqueness of positive solutions for FEP (1).

\section{Examples}

In the section, we give two concrete examples to illustrate our main results.

Example 5.1 Consider the FEP

$$
\left\{\begin{array}{l}
{ }^{C} D_{0^{+}}^{1.5} x(t)+\lambda f(t, x(t))=0, \quad 0<t<1, \\
x(0)-4 x^{\prime}(0)=0, \\
x(1)=\int_{0}^{1} \frac{1}{100}(s x(s))^{2} d s+\mu,
\end{array}\right.
$$

where

$$
f(t, x)= \begin{cases}\frac{t^{3}(x+1)^{\frac{3}{2}}}{\sqrt{6}}, & 0 \leq x \leq 1 \\ t^{3} \sqrt{\frac{x}{3}}, & x>1\end{cases}
$$

that is, $\alpha=1.5, a=1, b=4, g(x)=x^{2}$, and $k(t)=\frac{1}{100} t^{2}$. Evidently, $\frac{a}{a+b}<\alpha-1, f \in C([0,1] \times$ $\left.R_{+}, R_{+}\right), f(t, 0) \not \equiv 0, g \in C\left(R_{+}, R_{+}\right), k \in C\left([0,1], R_{+}\right)$, and $k \not \equiv 0$. It is easy check that (H1) holds and $g(x)$ is strictly increasing in $x \in[0,+\infty)$. Note that

$$
\limsup _{x \rightarrow+\infty} \frac{g(x)}{x}=+\infty, \quad \lim _{r \rightarrow 0} \frac{g(r)}{r}=0 .
$$

Therefore, $(\mathrm{H} 3)$ and $(\mathrm{H} 4)$ are satisfied. From Theorem 3.4 we obtain that there exist $\mu^{*}>0$ and $\lambda^{*}:\left(0, \mu^{*}\right] \rightarrow[0,+\infty)$ satisfying

$$
\lambda^{*}(\mu)>0, \quad 0 \leq \mu<\mu^{*}, \quad \lambda^{*}(\mu) \geq 0, \quad \lambda=\mu^{*},
$$

such that:

(i) FEP (32) has at least two positive solutions for $0<\mu<\mu^{*}$ and $0 \leq \lambda<\lambda^{*}(\mu)$;

(ii) FEP (32) has at least one positive solution for $0 \leq \mu<\mu^{*}$ and $\lambda=\lambda^{*}(\mu)$;

(iii) FEP (32) has no positive solutions for $0 \leq \mu \leq \mu^{*}, \lambda>\lambda^{*}(\mu)$ or $\mu>\mu^{*}, \lambda \geq 0$.

Next, we estimate the critical values $\mu^{*}>0$ and $\lambda^{*}(\mu)$ for $\mu \in\left[0, \mu^{*}\right]$. It is clear that

$$
0<r-g(r) \int_{0}^{1} k(s) d s \leq 150-g(150) \int_{0}^{1} k(s) d s=75, \quad 0<r<300,
$$


and

$$
f_{m}(r)=\max _{t \in[0,1]} f(t, r)= \begin{cases}\frac{(r+1)^{\frac{3}{2}}}{\sqrt{6}}, & 0 \leq r \leq 1, \\ \sqrt{\frac{r}{3}}, & r>1 .\end{cases}
$$

Take $r_{0}=150$; then $r_{0}-g\left(r_{0}\right) \int_{0}^{1} k(s) d s=75$. From Remark 3.5 we have $\mu^{*} \geq 75$ and, for $0 \leq \mu \leq 75$,

$$
\lambda^{*}(\mu) \geq \frac{(75-\mu) \sqrt{3} \Gamma(2.5)}{\sqrt{150}}=\frac{3 \sqrt{2 \pi}(75-\mu)}{40} .
$$

Example 5.2 Consider the FEP

$$
\left\{\begin{array}{l}
{ }^{C} D_{0^{+}}^{\alpha} x(t)+\lambda f(t, x)=0, \quad 0<t<1, \\
\frac{1}{9} x(0)-\frac{1}{3} x^{\prime}(0)=0, \\
x(1)=\int_{0}^{1} \frac{s x(s)}{1+x(s)} d s+\mu,
\end{array}\right.
$$

where $1.1<\alpha \leq 2$ and

$$
f(t, x)= \begin{cases}\frac{t}{3} x, & 0 \leq x \leq 1 \\ \frac{t}{6}(x+\sqrt{x}), & x>1\end{cases}
$$

that is, $a=\frac{1}{9}, b=1, g(x)=\frac{x}{1+x}$, and $k(t)=t$. Evidently, $\frac{a}{a+b}<\alpha-1, f \in C\left([0,1] \times R_{+}, R_{+}\right)$, $g \in C\left(R_{+}, R_{+}\right), k \in C\left([0,1], R_{+}\right)$, and $k \not \equiv 0$. It is easy check that (H1) and (H2) hold. For any $r \in(0,1)$,

for $0 \leq x \leq 1$, we have $f(t, r x)=\frac{t r}{3} x=r f(t, x)$;

if $x>1$ and $0<r x \leq 1$, then we have $f(t, r x)=\frac{t r}{3} x \geq \frac{r t}{3}\left(\frac{1}{2} x+\frac{1}{2} \sqrt{x}\right) \geq \frac{t r}{6}(x+\sqrt{x})=$ $r f(t, x)$;

if $x>1$ and $r x>1$, then we have $f(t, r x)=\frac{t}{6}(r x+\sqrt{r x}) \geq \frac{t r}{6}\left(x+\frac{1}{\sqrt{r}} \sqrt{x}\right) \geq r f(t, x)$, that is, $f(t, r x) \geq r f(t, x)$ for $x \in[0,+\infty)$ and $t \in[0,1]$. Hence, $f$ satisfies (H6). It is easy to check that $g$ satisfies (H7) and (H8). By Theorem 4.5 we obtain that for given $\mu>0$, there exists $\lambda^{*}(\mu)>0$ such that FEP (34) has a unique positive solution $x_{\lambda}$ for $\lambda \in\left[0, \lambda^{*}(\mu)\right)$ and has no positive solution for $\lambda \geq \lambda^{*}(\mu)$. Moreover, such a solution $x_{\lambda}$ satisfies the following properties:

(i) for any $u_{0} \in P$, setting $u_{n}(t)=\lambda \int_{0}^{1} G(t, s) f\left(s, u_{n-1}(s)\right) d s+e(t)\left(\int_{0}^{1} k(s) g\left(u_{n-1}(s)\right) d s+\right.$ $\mu), n=1,2, \ldots$, we have $\lim _{n \rightarrow \infty}\left\|u_{n}-x_{\lambda}\right\|=0$

(ii) $x_{\lambda}$ is increasing in $\lambda$ for $\lambda \in\left[0, \lambda^{*}(\mu)\right)$;

(iii) $x_{\lambda}$ is continuous with respect to $\lambda$ for $\lambda \in\left[0, \lambda^{*}(\mu)\right)$.

Next, we apply Theorem 4.6 to estimate the critical values $\lambda^{*}(\mu)$ for $\mu>0$. Note that

$$
F_{\infty}=\limsup _{x \rightarrow+\infty} \max _{t \in[0,1]} \frac{f(t, x)}{x}=\frac{1}{6} .
$$

Then $\lambda^{*}(\mu) \geq \frac{\Gamma(\alpha+1)}{2 F_{\infty}}=3 \Gamma(\alpha+1)$ for all $\mu>0$. 
Authors' contributions

All authors participated in drafting, revising, and commenting the manuscript. All authors read and approved the final manuscript.

\section{Author details}

'Department of Mathematics, Taiyuan Normal University, Taiyuan, 030012, P.R. China. ${ }^{2}$ School of Software, North University of China, Taiyuan, 030051, P.R. China.

\section{Acknowledgements}

The authors sincerely thank the reviewers for their valuable suggestions and useful comments. This research was supported by the NNSF of China (11361047), the University Natural Science Research Develop Foundation of Shanxi Province of China $(20111021,2013156)$, and Research Project Supported by Shanxi Scholarship Council of China (2013-102)

Received: 7 December 2015 Accepted: 27 January 2016 Published online: 10 February 2016

\section{References}

1. Kilbas, AA, Srivastava, HM, Trujillo, JJ: Theory and Applications of Fractional Differential Equations. Elsevier, Amsterdam (2006)

2. Podlubny, I: Fractional Differential Equations. Mathematics in Science and Engineering. Academic Press, New York (1999)

3. Baleanu, D, Diethelm, K, Scalas, E, Trujillo, JJ: Fractional Calculus Models and Numerical Methods. Series on Complexity, Nonlinearity and Chaos. World Scientific, Boston (2012)

4. Lakshmikantham, V, Leela, S, Devi, JV: Theory of Fractional Dynamic Systems. Cambridge Scientific Publishers, Cambridge (2009)

5. Lakshmikantham, V, Vatsala, AS: Basic theory of fractional differential equations. Nonlinear Anal. 69, $2677-2682$ (2008)

6. Benchohra, M, Henderson, J, Ntouyas, SK, Ouahab, A: Existence results for fractional order functional differential equations with infinite delay. J. Math. Anal. Appl. 338, 1340-1350 (2008)

7. Jleli, M, Samet, B: Existence of positive solutions to an arbitrary order fractional differential equation via a mixed monotone operator method. Nonlinear Anal. 20, 367-376 (2015)

8. Agarwal, RP, Benchohra, M, Hamani, S: Boundary value problems for fractional differential equations. Adv. Stud. Contemp. Math. 16, 181-196 (2008)

9. Benchohra, M, Graef, JR, Hamani, S: Existence results for boundary value problems with nonlinear fractional differential equations. Appl. Anal. 87, 851-863 (2008)

10. Benchohra, M, Hamani, S, Ntouyas, SK: Boundary value problems for differential equations with fractional order. Surv. Math. Appl. 3, 1-12 (2008)

11. Sun, B, Yang, A, Ge, WG: Successive iteration and positive solutions for some second-order three-point $p$-Laplacian boundary value problems. Math. Comput. Model. 50, 344-350 (2009)

12. Al-Refai, M, Haiji, MA: Monotone iterative sequences for nonlinear boundary value problems of fractional order Nonlinear Anal. 74(3), 531-3539 (2011)

13. Jiang, DQ, Yuan, C: The positive properties of the Green function for Dirichlet-type boundary value problems of nonlinear fractional differential equations and its application. Nonlinear Anal. 72, 710-719 (2010)

14. Jankowski, T: Boundary problems for fractional differential equations. Appl. Math. Lett. 28, 14-19 (2014)

15. Lin, L, Liu, X, Fang, H: Method of upper and lower solutions for fractional differential equations. Electron. J. Differ. Equ. $2012,100(2012)$

16. Liu, Z, Sun, J, Szántá, I: Monotone iterative technique for Riemann-Liouville fractional integro-differential equations with advanced arguments. Results Math. 63, 1277-1287 (2013)

17. Wang, GT, Baleanu, D, Zhang, L: Monotone iterative method for a class of nonlinear fractional differential equations. Fract. Calc. Appl. Anal. 15, 244-252 (2012)

18. Zhou, WX, Chu, YD, Baleanu, D: Uniqueness and existence of positive solutions for a multi-point boundary value problem of singular fractional differential equations. Adv. Differ. Equ. 2013, 114 (2013)

19. Ahmad, B, Nieto, JJ: Existence results for nonlinear boundary value problems of fractional integro-differential equations with integral boundary conditions. Bound. Value Probl. 2009, 708576 (2009). doi:10.1155/2009/708576

20. Ahmad, B, Nieto, JJ, Alsaedi, A: Existence and uniqueness of solutions for nonlinear fractional differential equations with non-separated type integral boundary conditions. Acta Math. Sci. 31(6), 2122-2130 (2011)

21. Ahmad, B, Ntouyas, SK, Alsaedi, A: New existence results for nonlinear fractional differential equations with three-point integral boundary conditions. Adv. Differ. Equ. 2011, 107384 (2011). doi:10.1155/2011/107384

22. Feng, MQ, Ji, D, Ge, WG: Positive solutions for a class of boundary-value problem with integral boundary conditions in Banach spaces. J. Comput. Appl. Math. 222(2), 351-363 (2008)

23. Yang, WG: Positive solutions for a coupled system of nonlinear fractional differential equations with integral boundary conditions. Comput. Math. Appl. 63(1), 288-297 (2012)

24. Khan, RA, Rehman, MU, Henderson, J: Existence and uniqueness of solutions for nonlinear fractional differential equations with integral boundary conditions. Fract. Differ. Calc. 1(1), 29-43 (2011)

25. Feng, MQ, Zhang, XM, Ge, WG: New existence results for higher-order nonlinear fractional differential equation with integral boundary conditions. Bound. Value Probl. 2011, 720702 (2011). doi:10.1155/2011/720702

26. Liu, XP, Jia, M: Multiple solutions for fractional differential equations with nonlinear boundary conditions. Comput. Math. Appl. 59(8), 2880-2886 (2010)

27. Jin, JF, Liu, XP, Jia, M: Existence of positive solutions for singular fractional differential equations with integral boundary conditions. Electron. J. Differ. Equ. 2012, 63 (2012)

28. Bai, ZB: Eigenvalue intervals for a class of fractional boundary value problem. Comput. Math. Appl. 64(10), 3253-3257 (2012)

29. Jiang, W: Eigenvalue interval for multi-point boundary value problems of fractional differential equations. Appl. Math. Comput. 219(9), 4570-4575 (2013) 
30. Sun, S, Zhao, Y, Han, Z, Liu, J: Eigenvalue problem for a class of nonlinear fractional differential equations. Ann. Funct. Anal. 4(1), 25-39 (2013)

31. Zhai, $C B, X u, L$ : Properties of positive solutions to a class of four-point boundary value problem of Caputo fractional differential equations with a parameter. Commun. Nonlinear Sci. Numer. Simul. 19, 2820-2827 (2014)

32. Zhang, XG, Liu, LS, Wiwatanapataphee, B, Wu, YH: The eigenvalue for a class of singular $p$-Laplacian fractional differential equations involving the Riemann-Stieltjes integral boundary condition. Appl. Math. Comput. 235, 412-422 (2014)

33. Wang, GT, Liu, SY, Zhang, LH: Eigenvalue problem for nonlinear fractional differential equations with integral boundary conditions. Abstr. Appl. Anal. 2014, 916260 (2014)

34. Jia, M, Liu, XP: The existence of positive solution for fractional differential equations with integral and disturbance parameter in boundary conditions. Abstr. Appl. Anal. 2014, 131548 (2014)

35. Guo, DJ, Lakshmikantham, V: Nonlinear Problems in Abstracts Cone. Academic Press, New York (1988)

36. Deimling, K: Nonlinear Functional Analysis. Springer, Berlin (1985)

37. Wang, WX, Liu, XL, Shi, PP: Nonlinear sum operator equations with a parameter and application to second-order three-point BVPs. Abstr. Appl. Anal. 2014, 259016 (2014)

38. Feng, WY, Zhang, G: New fixed point theorems on order intervals and their applications. Fixed Point Theory Appl. (2015). doi:10.1186/s13663-015-0467-2

39. Zhang, G, Cheng, SS: Positive periodic solutions of coupled delay differential systems depending on two parameters. Taiwan. J. Math. 8(4), 639-652 (2004)

\section{Submit your manuscript to a SpringerOpen ${ }^{\mathcal{O}}$ journal and benefit from:}

- Convenient online submission

- Rigorous peer review

- Immediate publication on acceptance

Open access: articles freely available online

- High visibility within the field

- Retaining the copyright to your article 